\title{
Trajectory Design Employing Convex Optimization for Landing on Irregularly Shaped Asteroids
}

\author{
Robin Pinson (presenter) \\ NASA Marshall Space Flight Center \\ Ping Lu \\ San Diego State University
}

September 14, 2016

SPACE 2016 Long Beach, CA 


\section{Goal}

- Goal: Design an optimal powered descent trajectory on-board the spacecraft in order to softly land on an irregularly shaped asteroid.

- Algorithm needs to be autonomous, reliable, robust, and efficient.

- Designing on-board facilitates an easy change of parameters.

- Convex optimization is efficient and reliable.

- Guarantees global minimum in a finite number of steps, if the problem is feasible.

- Subclasses include Second Order Cone Programming (SOCP).

- Can convex optimization be used to design the asteroid powered descent trajectory? 


\section{Original Problem Formulation}

- Asteroid powered descent propellant optimal problem is nonlinear and nonconvex.

$$
\begin{gathered}
\min -m\left(t_{f}\right) \\
\text { s.t. } \dot{\vec{r}}=\vec{v}, \dot{\vec{v}}=\frac{\vec{T}}{m}-2 \vec{\omega} \times \vec{v}-\dot{\vec{\omega}} \times \vec{r}-\vec{\omega} \times(\vec{\omega} \times \vec{r})+\nabla U(\vec{r}), \dot{m}=-\frac{1}{v_{e x}}\|\vec{T}\| \\
T_{\min } \leq\|\vec{T}\| \leq T_{\max },\left\|\vec{r}-\vec{r}_{f}\right\| \cos \theta-\left(\vec{r}-\vec{r}_{f}\right)^{T} \hat{n} \leq 0, m \geq m_{d r y} \\
\vec{r}(0)=\vec{r}_{0}, \vec{v}(0)=\vec{v}_{0}, m(0)=m_{\text {wet }}, \vec{r}\left(t_{f}\right)=\vec{r}_{f}, \vec{v}\left(t_{f}\right)=\vec{v}_{f}, t_{f} \text { given }
\end{gathered}
$$

- Fixed final time two point value boundary problem

- State: $\vec{r}, \vec{v}, m$

- Control: $\vec{T}$

- Highlighted terms are not permissible for a convex optimization problem.

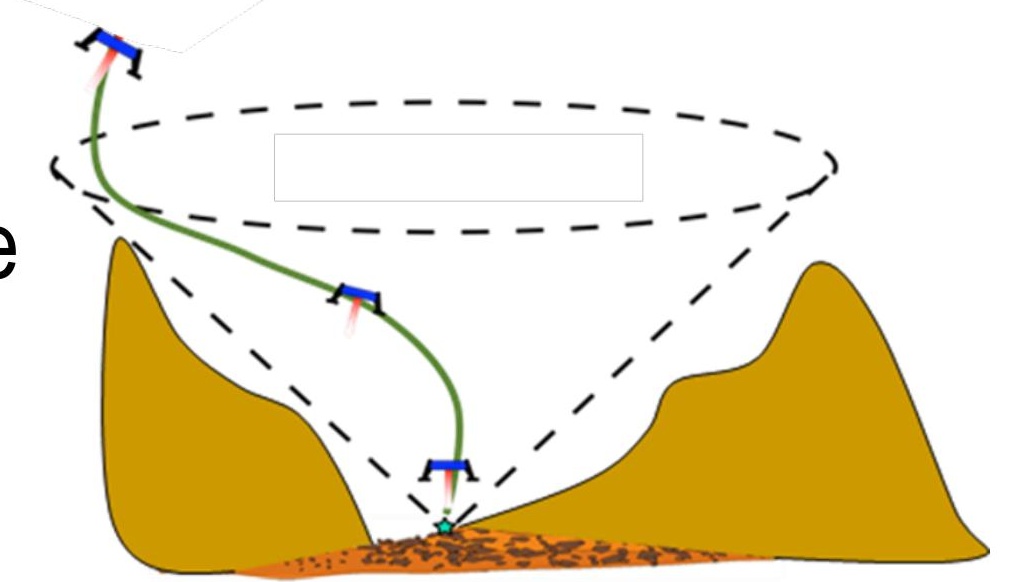




\section{Problem Relaxation}

Relax the problem by introducing a slack variable, $T_{m}$.

Original Problem

$\min -m\left(t_{f}\right)$

s.t. $\dot{\vec{r}}=\vec{v}$

$\dot{\vec{v}}=\frac{\vec{T}}{m}-2 \vec{\omega} \times \vec{v}-\dot{\vec{\omega}} \times \vec{r}$

$-\vec{\omega} \times(\vec{\omega} \times \vec{r})+\nabla U(\vec{r})$

$\dot{m}=-\frac{1}{v_{e x}}\|\vec{T}\|$

$T_{\min } \leq\|\vec{T}\| \leq T_{\max }$

$\left\|\vec{r}-\vec{r}_{f}\right\| \cos \theta-\left(\vec{r}-\vec{r}_{f}\right)^{T} \hat{n} \leq 0$

$m \geq m_{d r y}$

$\vec{r}(0)=\vec{r}_{0}, \vec{v}(0)=\vec{v}_{0}, m(0)=m_{\text {wet }}$

$\vec{r}\left(t_{f}\right)=\vec{r}_{f}, \vec{v}\left(t_{f}\right)=\vec{v}_{f}, t_{f}$ given
Relaxed Problem

$$
\min -m\left(t_{f}\right)
$$

s.t. $\dot{\vec{r}}=\vec{v}$

$$
\begin{aligned}
\dot{\vec{v}} & =\frac{\vec{T}}{m}-2 \vec{\omega} \times \vec{v}-\dot{\vec{\omega}} \times \vec{r} \\
& -\vec{\omega} \times(\vec{\omega} \times \vec{r})+\nabla U(\vec{r})
\end{aligned}
$$

$\dot{m}=-\frac{1}{v_{e x}} T_{m}$ Slack Variable

\section{$\vec{T} \| \leq T_{m} \quad$ New Constraint}

$$
\begin{aligned}
& T_{\min } \leq T_{m} \leq T_{\max } \\
& \left\|\vec{r}-\vec{r}_{f}\right\| \cos \theta-\left(\vec{r}-\vec{r}_{f}\right)^{T} \hat{n} \leq 0 \\
& m \geq m_{d r y} \\
& \vec{r}(0)=\vec{r}_{0}, \vec{v}(0)=\vec{v}_{0}, m(0)=m_{\text {wet }} \\
& \vec{r}\left(t_{f}\right)=\vec{r}_{f}, \vec{v}\left(t_{f}\right)=\vec{v}_{f}, t_{f} \text { given }
\end{aligned}
$$

Proved the optimal solution of the relaxed problem is the optimal solution of the original. 


\section{Irregularly Shaped Asteroid Gravity Models}

- 4x4 Spherical Harmonics Model

- Maximum Order and Degree 4

- No symmetry nor coordinate system location and alignment assumptions.

- High accuracy outside the Brillouin sphere.

- Not valid inside the Brillouin sphere.

- Interior spherical Bessel gravity model

- Valid inside the entire Brillouin sphere.

- Error less than $10 \%$ for the binary asteroid Castalia

- Published in 2014 by Takahashi and Scheeres.

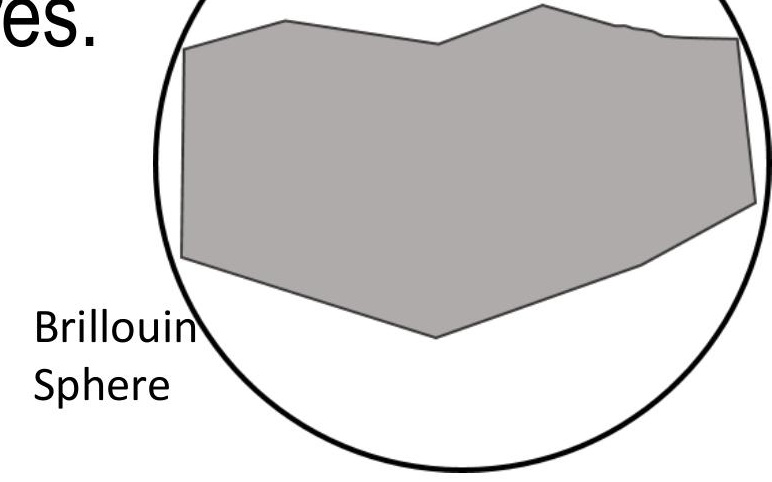




\section{4x4 Bessel}

- 4x4 spherical harmonics gravity model outside the Brillouin sphere.

- Interior spherical Bessel gravity model inside the Brillouin sphere.

- Both models are summation series.

- Highly nonlinear in terms of spacecraft position vector.

- Computational similarities between the models allows for easy transition between the models. 


\section{Successive Solution Method}

- Solve a series of convex optimization problems.

- Equations of motion can be arranged as:

$$
\dot{\vec{x}}^{(k)}=A\left(\vec{r}^{(k-1)}\right) \vec{x}^{(k)}+B \vec{u}^{(k)}+c\left(\vec{r}^{(k-1)}\right)
$$

- $A$ and $c$ are evaluated using the previous solution (k-1).

- In the $(k)^{\text {th }}$ iteration, dynamics are linear and time varying.

- Iterations continue until two successive trajectories are within a set tolerance.

- This is not the same as conventional linearization, as there are no approximations in the final iteration.

- Dominant gravity term is placed in A, with the higher order gravity terms in $\mathrm{c}$. 


\section{Successive Solution Method: A, B, C}

$$
\begin{aligned}
& A=\left[\begin{array}{ccccccc}
0 & 0 & 0 & 1 & 0 & 0 & 0 \\
0 & 0 & 0 & 0 & 1 & 0 & 0 \\
0 & 0 & 0 & 0 & 0 & 1 & 0 \\
\omega^{2}+d o m & 0 & 0 & 0 & 2 \omega & 0 & 0 \\
0 & \omega^{2}+d o m & 0 & -2 \omega & 0 & 0 & 0 \\
0 & 0 & d o m & 0 & 0 & 0 & 0 \\
0 & 0 & 0 & 0 & 0 & 0 & 0
\end{array}\right]^{(k-1)} \quad B=\left[\begin{array}{cccc}
0 & 0 & 0 & 0 \\
0 & 0 & 0 & 0 \\
0 & 0 & 0 & 0 \\
1 & 0 & 0 & 0 \\
0 & 1 & 0 & 0 \\
0 & 0 & 1 & 0 \\
0 & 0 & 0 & -\frac{1}{v_{e x}}
\end{array}\right] \\
& c=\left[\begin{array}{c}
0 \\
0 \\
0 \\
\frac{\partial U}{\partial r_{x}}-d o m \\
\frac{\partial U}{\partial r_{y}}-d o m \\
\frac{\partial U}{\partial r_{z}}-d o m \\
0
\end{array}\right]^{(k-1)} \\
& d o m= \begin{cases}-\frac{\mu}{r^{3}} & 4 \times 4 \\
\alpha_{0,0} j_{1}\left(\frac{\alpha_{0,0} r}{R_{b}}\right) \bar{A}_{0,0,0}+\alpha_{1,0} j_{1}\left(\frac{\alpha_{1,0} r}{R_{b}}\right) \bar{A}_{1,0,0}+\alpha_{2,0} j_{1}\left(\frac{\alpha_{2,0} r}{R_{b}}\right) \bar{A}_{2,0,0} & \text { Bessel }\end{cases}
\end{aligned}
$$




\section{Additional Techniques}

- Change of Variables

- Discretization

- Continuous equation of motion turned into discrete equality constraints.

- Scaling

- Final optimization problem is convex.

- Linear equality constraints

- Convex inequality constraints

- Inequality constraints are second order cone.

- Actually a SOCP 


\section{Optimal Flight Time}

- Desire to find the optimal flight time corresponding to smallest propellant usage.

- Propellant usage is unimodal with respect to flight time.

- Create an outer optimization loop using Brent's method to optimize the flight time.

- Use $d t=10.0 \mathrm{sec}$ to find the optimal flight time. Design the final trajectory with $d t=2.0 \mathrm{sec}$.

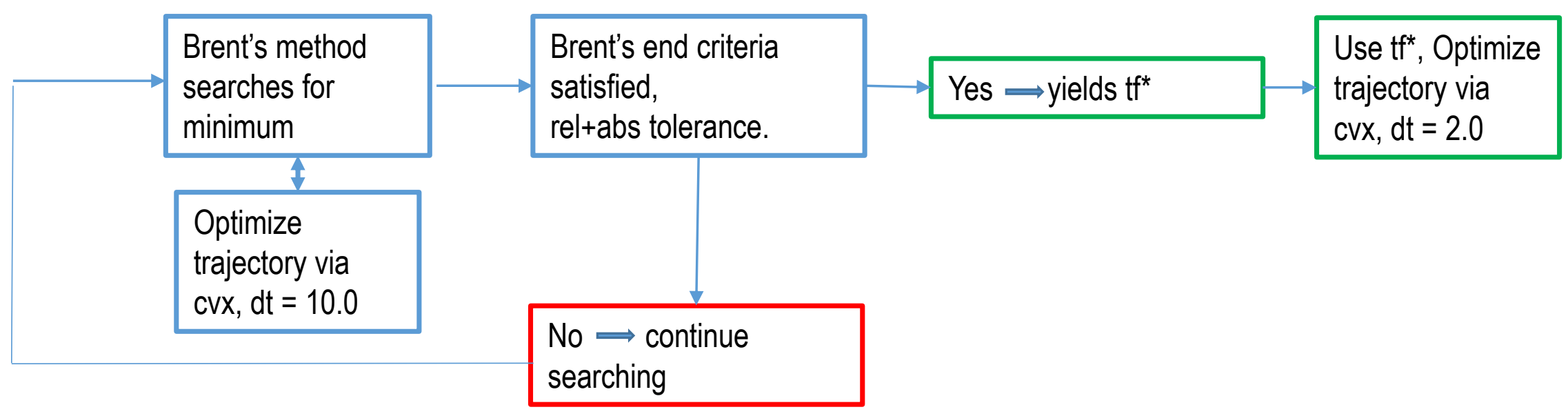




\section{Simulation Parameters}

- Asteroid Castalia

- Period $4.07 \mathrm{hr}$ along $+Z$ axis

- Three Landing Sites

- Spacecraft:

- Mass $1400 \mathrm{~kg}$

$\diamond 400 \mathrm{~kg}$ propellant

- Thrust $80 \mathrm{~N}-20 \mathrm{~N}$

- Initial Conditions

- 1000 m altitude

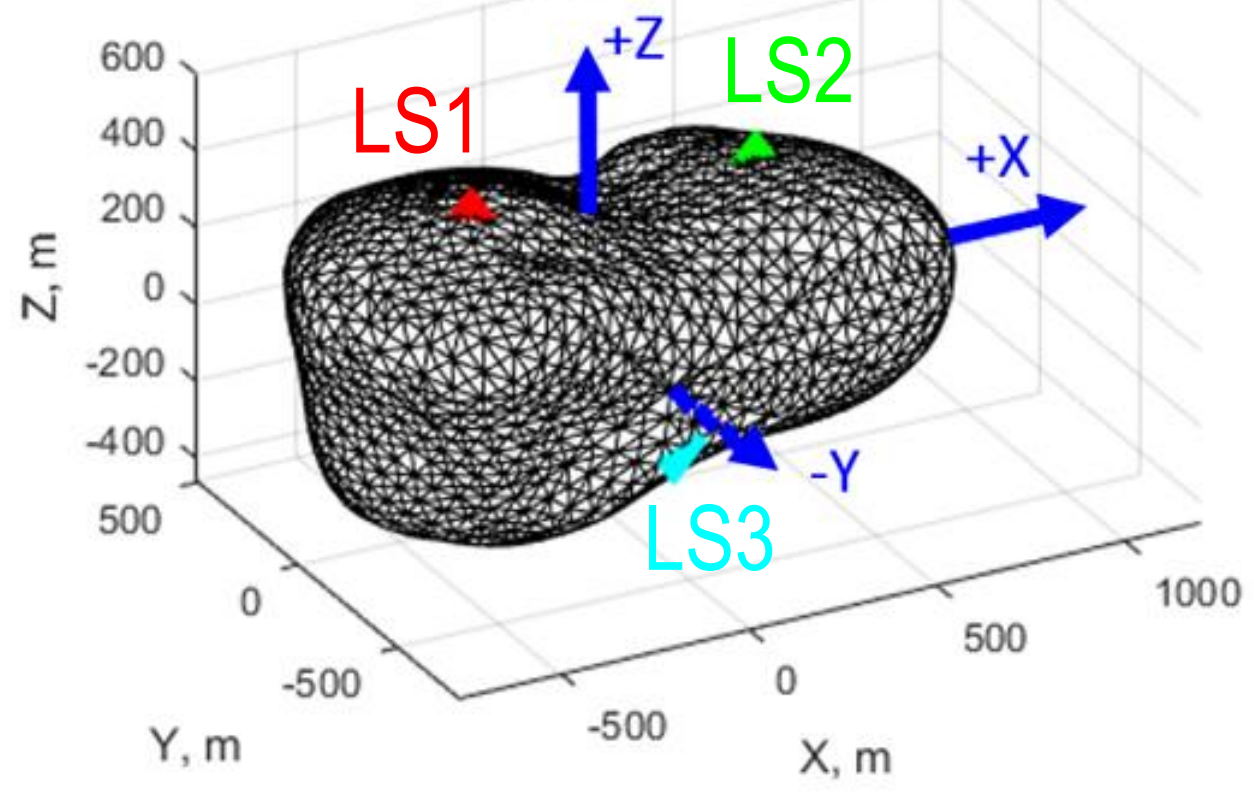

- Out of plane and uprange position and velocity components.

- Using CVX, a publically available Matlab based convex optimization program. 


\section{Flight Time Parameter Sweep}

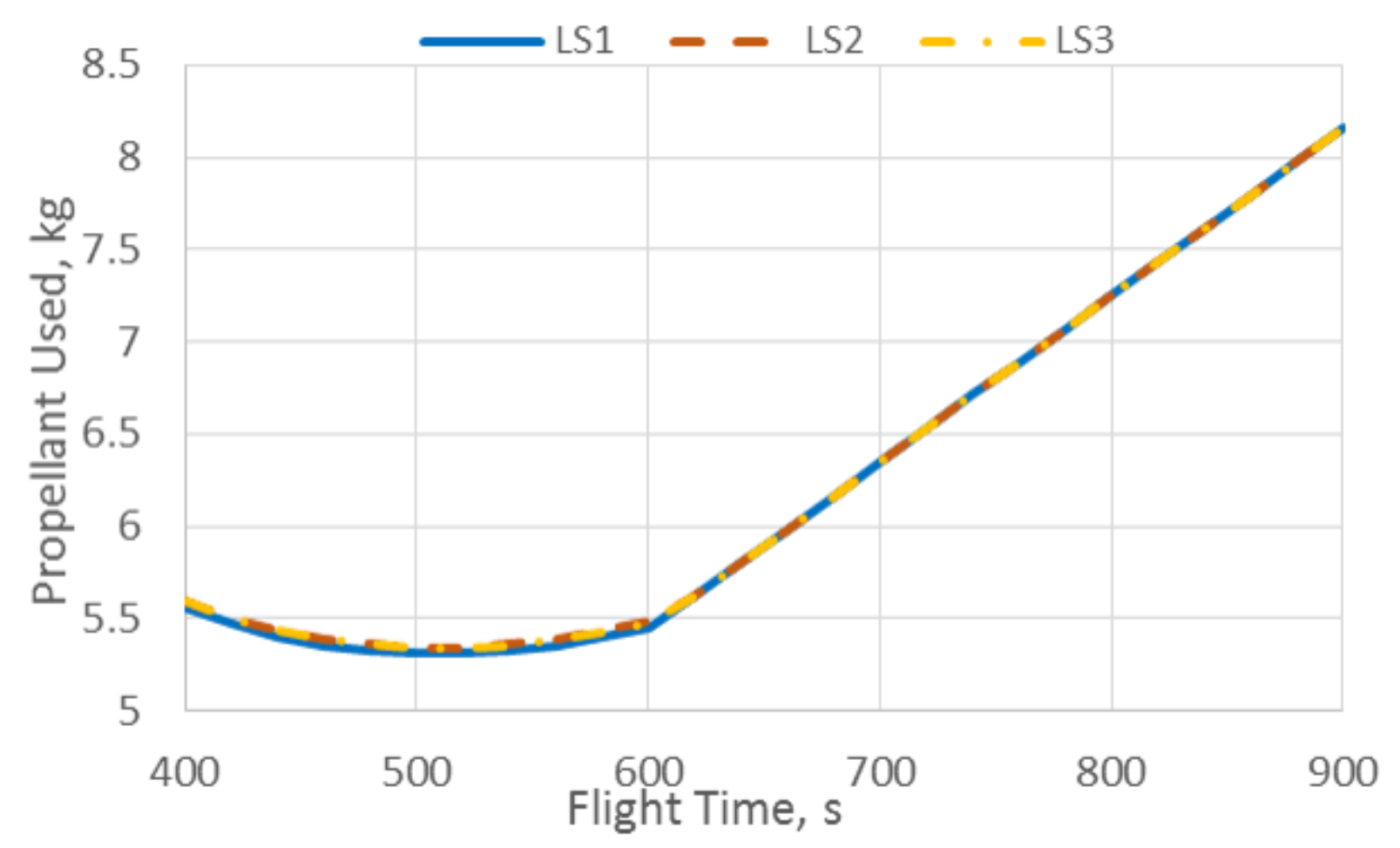

- Typically 3 iterations required in the successive solution method.

- Range 3-7

- Low number of iterations demonstrates stability in the successive solution methodology. 


\section{Inner Loop Trajectory Design}

- 400 Second Flight Time landing at LS3

- Thrust profile follows the traditional bang-bang.
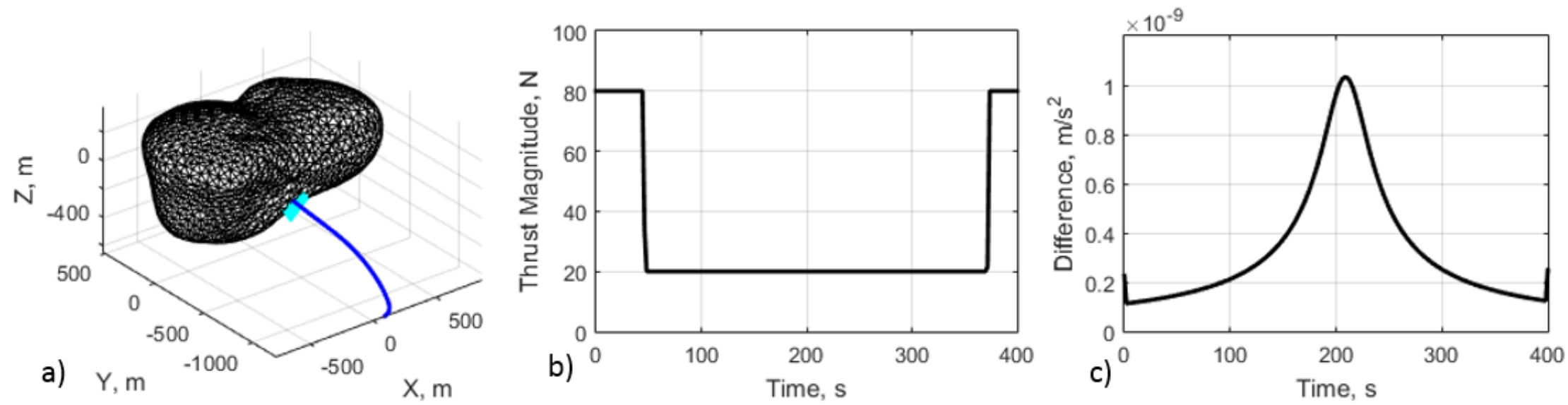


\section{Optimal Flight Time Optimal Propellant Trajectory}

- Combined outer and inner loop executions took 2.2 - 2.5 minutes.

\begin{tabular}{|l|l|l|l|} 
& $\begin{array}{l}\text { Optimal Flight } \\
\text { Time, s }\end{array}$ & $\begin{array}{l}\text { Propellant } \\
\text { Used, kg }\end{array}$ & $\begin{array}{l}\text { Number of Inner } \\
\text { Loop } \\
\text { Executions }\end{array}$ \\
\hline LS1 & 512.86 & 5.31 & 7 \\
\hline LS2 & 512.27 & 5.34 & 7 \\
\hline LS3 & 513.35 & 5.34 & 7 \\
\hline
\end{tabular}




\section{Optimal Flight Time Optimal Propellant Trajectory Parameters}

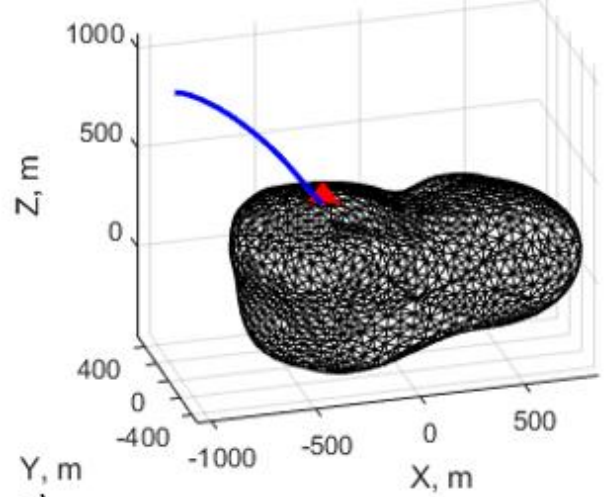

a) LS1

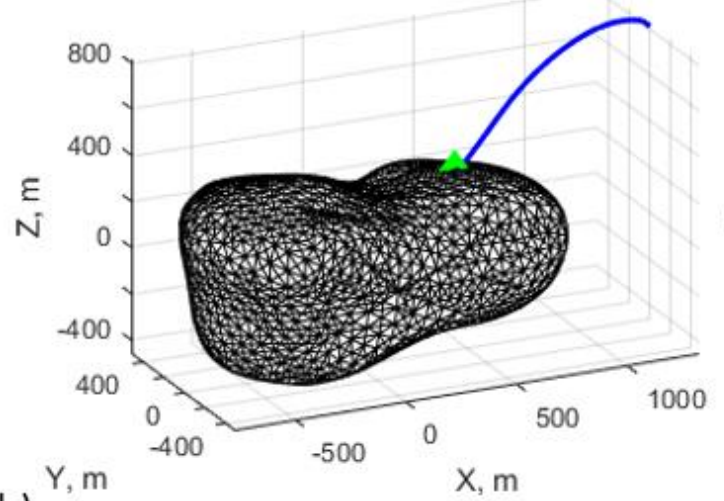

b) $\stackrel{Y, m}{L S 2}$

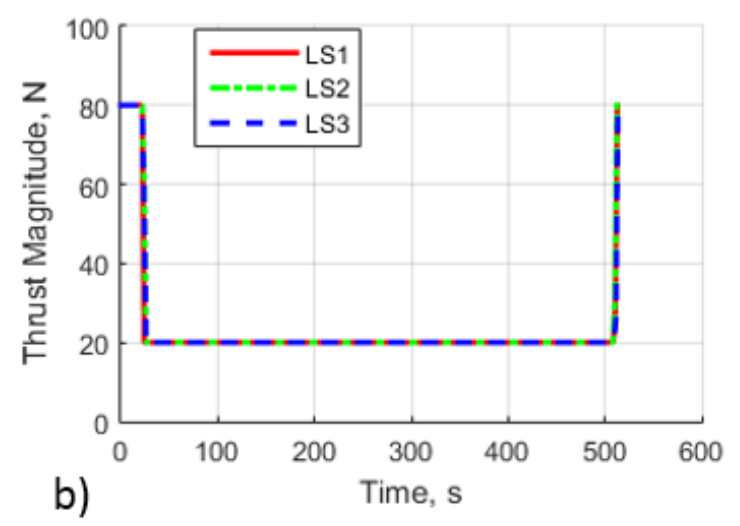

Thrust Magnitude

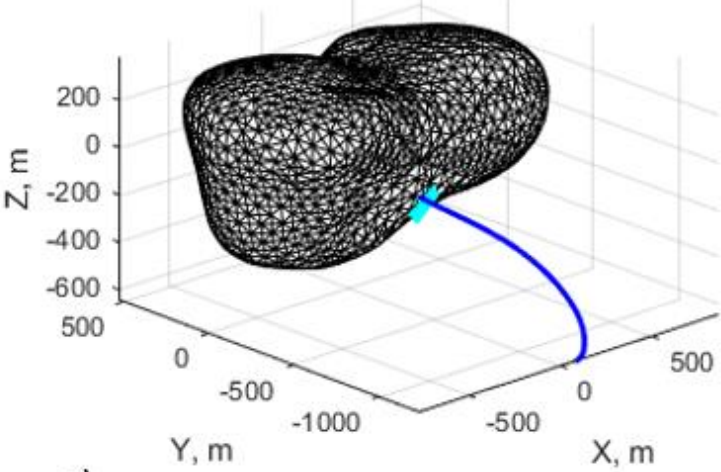

c) LS3

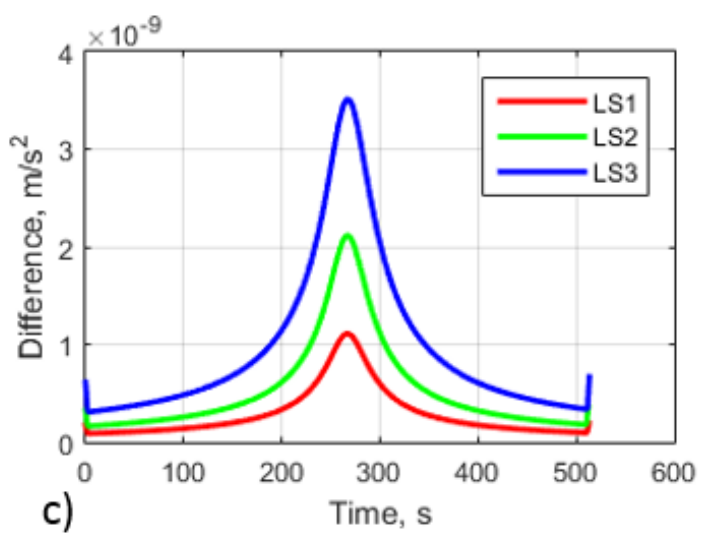

Slack Variable Check

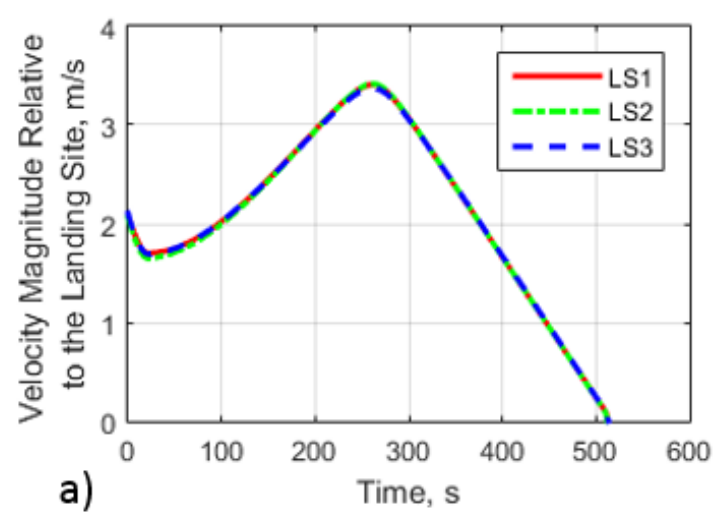

Velocity Magnitude 


\section{Glide Slope Constraint}

- Glide slope constraint: Constrains the vehicle to fly inside a cone around the landing site.

$$
\text { - }\left\|\vec{r}-\vec{r}_{f}\right\| \cos \theta-\left(\vec{r}-\vec{r}_{f}\right)^{T} \hat{n} \leq 0
$$

- Near the landing site the vehicle must match the landing site velocity to rotate with the landing site.

- Low thrust of the vehicle $(80 \mathrm{~N})$ prohibits this.

- Alternate solutions for a 10 deg cone:

- Increase max thrust to $320 \mathrm{~N}$

- Enforce the constraint for all, but the last 6 seconds.

$\diamond$ Flies slightly outside the cone near the surface.

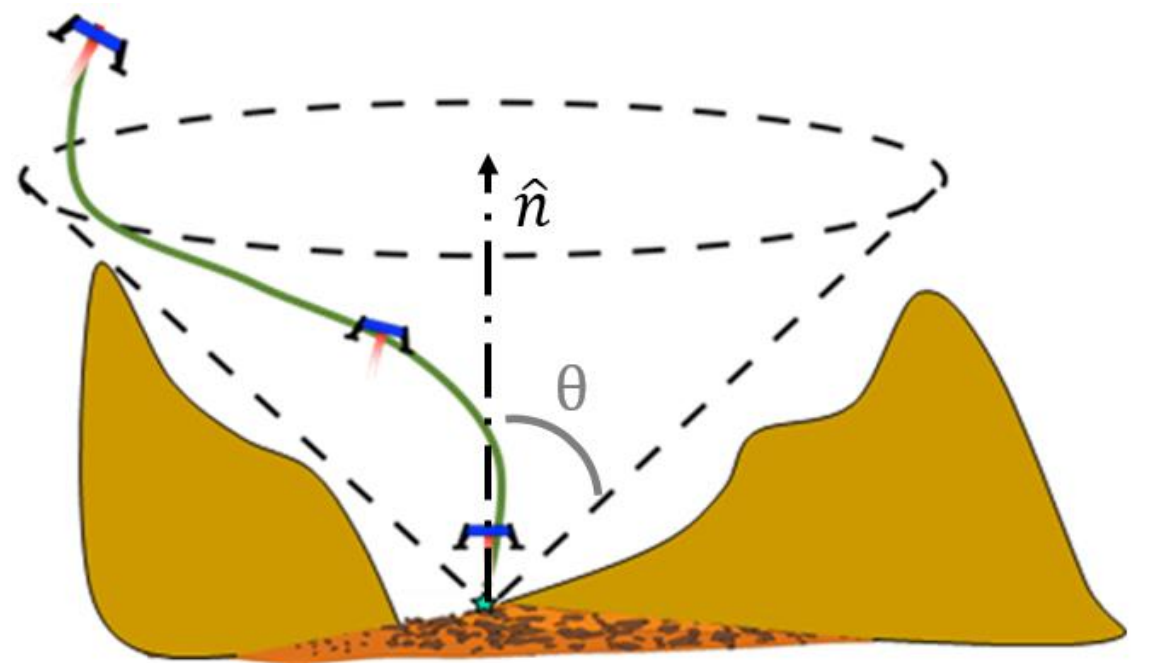




\section{Glide Slope Results}

- LS2 500 second flight time.

- 10 degree cone enforced.
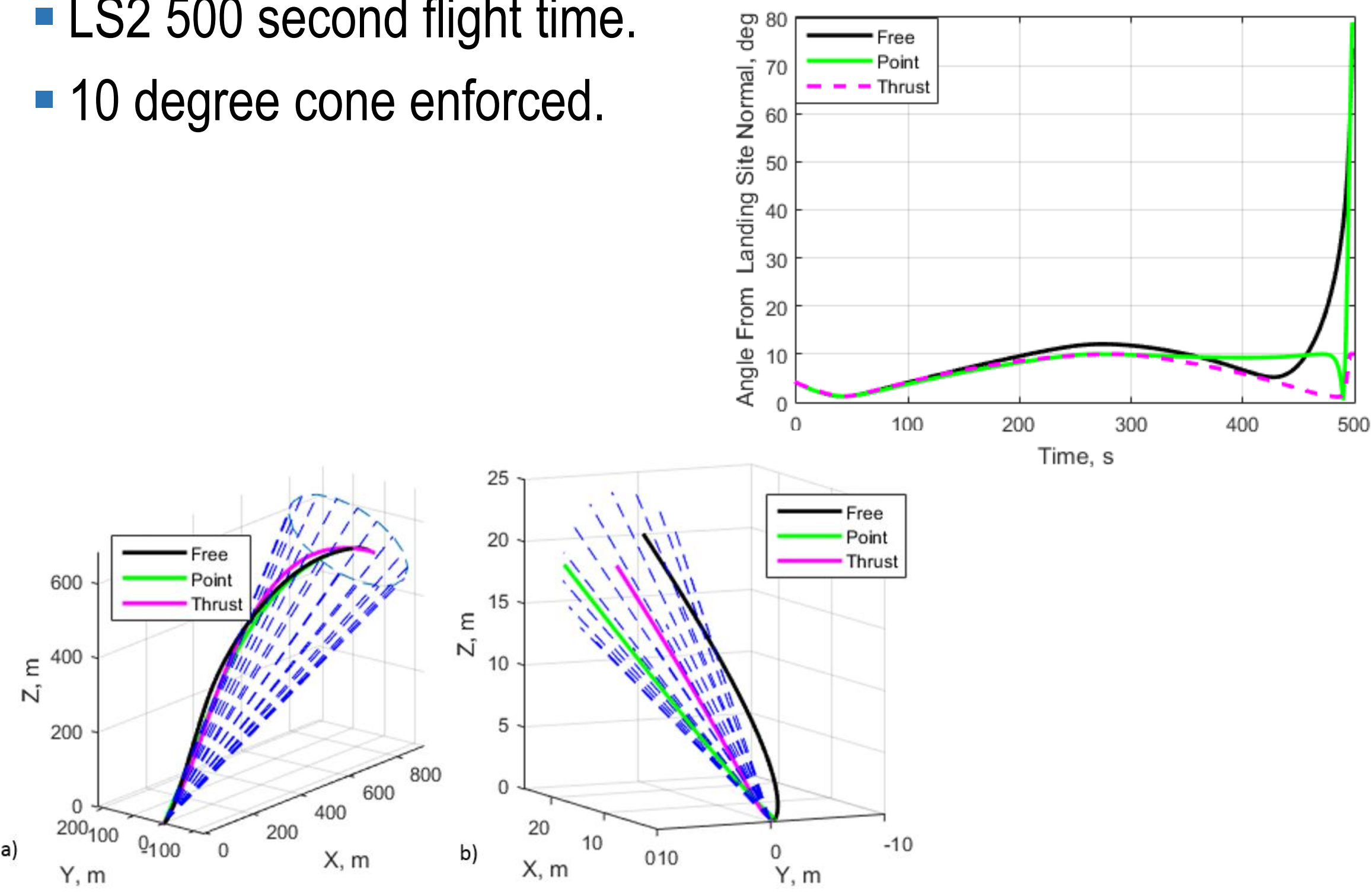


\section{Conclusions}

- Asteroid powered descent trajectory design can be formulated as a convex optimization problem.

- Successive solution methodology is the key to handling a nonlinear gravity model.

- Formulated algorithm handles a wide range of parameters successfully.

- Flight time optimization is completed in an outer loop with Brent's method.

- Inclusion of additional trajectory constraints in the algorithm is feasible.

- Viable algorithm for rapidly designing asteroid powered descent trajectories autonomously on-board the spacecraft for use in a variety of guidance algorithms. 
BACK-UP 


\section{Convex Optimization and SOCP Formulation}

- Optimization problem formulation $\min g(x)$

$$
\begin{aligned}
\text { s.t. } f_{i}(x) & \leq 0 i=1, \ldots, m \\
h_{j}(x) & =0 j=1, \ldots, p
\end{aligned}
$$

- Convex Optimization

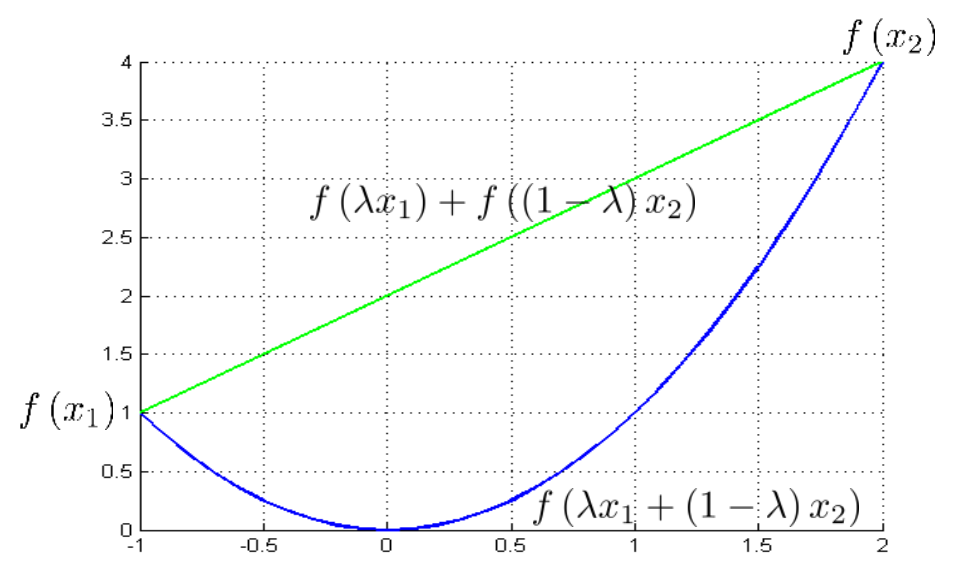

- $g(x)$ and $f_{i}(x)$ are convex functions.

- $h_{j}(x)$ is linear.

- Convex Function: $f\left(\lambda x_{1}+(1-\lambda) x_{2}\right) \leq f\left(\lambda x_{1}\right)+f\left((1-\lambda) x_{2}\right)$

- Second Order Cone Program (SOCP)

- Subset of convex optimization

- $g(x)$ and $h_{j}(x)$ are linear functions.

- $f_{i}(x)$ is second order cone.

- Second order Cone: $\|M x+d\|_{2} \leq c$

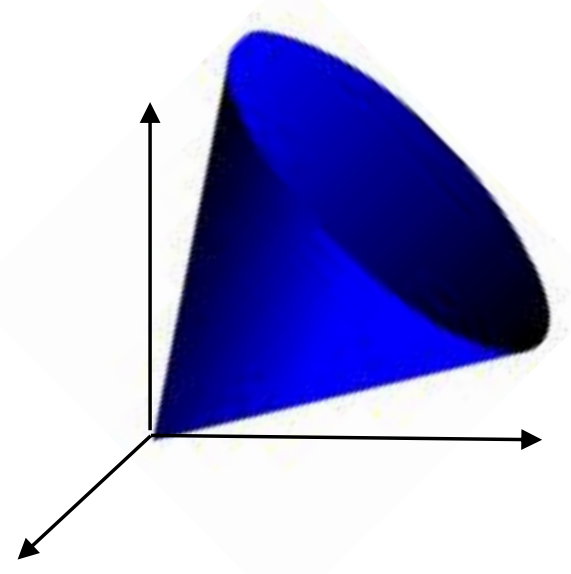




\section{Spherical Harmonics Gravity Model}

- Fidelity determined by the coefficients and the number of terms in the summation series.

$$
N= \begin{cases}2 & 2 \times 2 \\ 4 & 4 \times 4\end{cases}
$$

- Partial of the gravitational potential with respect to the position vector in spherical coordinates:

- $\nabla U(\vec{r})$ with respect to the Cartesian coordinate system:

$$
\begin{aligned}
& \frac{\partial U}{\partial r_{x}}=\frac{\partial U}{\partial r} \frac{\partial r}{\partial x}+\frac{\partial U}{\partial \delta} \frac{\partial \delta}{\partial x}+\frac{\partial U}{\partial \lambda} \frac{\partial \lambda}{\partial x} \\
& \frac{\partial U}{\partial r_{y}}=\frac{\partial U}{\partial r} \frac{\partial r}{\partial y}+\frac{\partial U}{\partial \delta} \frac{\partial \delta}{\partial y}+\frac{\partial U}{\partial \lambda} \frac{\partial \lambda}{\partial y} \\
& \frac{\partial U}{\partial r_{z}}=\frac{\partial U}{\partial r} \frac{\partial r}{\partial z}+\frac{\partial U}{\partial \delta} \frac{\partial \delta}{\partial z}+\frac{\partial U}{\partial \lambda} \frac{\partial \lambda}{\partial z}
\end{aligned}
$$

$P_{l, m}$ associated Legendre function

$l, m$ order, degree

$r, \delta, \lambda$ radius, latitude, longitude

$\frac{\partial U}{\partial r}=\sum_{l=0}^{N} \sum_{m=0}^{l}-(l+1) \frac{\mu}{r^{2}}\left(\frac{r_{0}}{r}\right)^{l} P_{l, m}[\sin \delta]\left\{C_{l, m} \cos (m \lambda)+S_{l, m} \sin (m \lambda)\right\}$

$\frac{\partial U}{\partial \delta}=\sum_{l=0}^{N} \sum_{m=0}^{l} \frac{\mu}{r}\left(\frac{r_{0}}{r}\right)^{l}\left\{C_{l, m} \cos (m \lambda)+S_{l, m} \sin (m \lambda)\right\} \frac{\partial P_{l, m}[\sin \delta]}{\partial \delta}$ $\frac{\partial U}{\partial \lambda}=\sum_{l=0}^{N} \sum_{m=0}^{l} \frac{\mu}{r}\left(\frac{r_{0}}{r}\right)^{l} m P_{l, m}[\sin \delta]\left\{-C_{l, m} \sin (m \lambda)+S_{l, m} \cos (m \lambda)\right\}$

- Partial of the position vector in spherical coordinate system with respect to the Cartesian:

$$
\begin{gathered}
\frac{\partial r}{\partial x}=\frac{r_{x}}{r}, \frac{\partial r}{\partial y}=\frac{r_{y}}{r}, \frac{\partial r}{\partial z}=\frac{r_{z}}{r} \\
\frac{\partial \delta}{\partial x}=\frac{-r_{x} r_{z}}{r^{2} \sqrt{r_{x}^{2}+r_{y}^{2}}}, \frac{\partial \delta}{\partial y}=\frac{-r_{y} r_{z}}{r^{2} \sqrt{r_{x}^{2}+r_{y}^{2}}}, \frac{\partial \delta}{\partial z}=\frac{1}{\sqrt{r_{x}^{2}+r_{y}^{2}}}\left(1-\frac{r_{z}^{2}}{r^{2}}\right) \\
\frac{\partial \lambda}{\partial x}=\frac{-r_{y}}{r_{x}^{2}+r_{y}^{2}}, \frac{\partial \lambda}{\partial x}=\frac{r_{x}}{r_{x}^{2}+r_{y}^{2}}, \frac{\partial \lambda}{\partial z}=0
\end{gathered}
$$




\section{Interior spherical Bessel Gravity Model}

- Summation Series: $l_{\max }=2, n_{\max }=5, m_{\max }=5$

$$
\begin{aligned}
& \frac{\partial U}{\partial r_{x}}=\frac{\mu}{R_{b}} \sum_{l=0}^{l_{\max }} \sum_{n=0}^{n_{\max }} \sum_{m=0}^{n} R e\left[\frac{\partial}{\partial x}\left(\bar{\beta}_{n, m}\left(\alpha_{l, n}\right)\right)\right] \bar{A}_{l, n, m}+\operatorname{Im}\left[\frac{\partial}{\partial x}\left(\bar{\beta}_{n, m}\left(\alpha_{l, n}\right)\right)\right] \bar{B}_{l, n, m} \\
& \frac{\partial U}{\partial r_{y}}=\frac{\mu}{R_{b}} \sum_{l=0}^{l_{\max }} \sum_{n=0}^{m_{m a x}} \sum_{m=0}^{n} R e\left[\frac{\partial}{\partial y}\left(\bar{\beta}_{n, m}\left(\alpha_{l, n}\right)\right)\right] \bar{A}_{l, n, m}+\operatorname{Im}\left[\frac{\partial}{\partial y}\left(\bar{\beta}_{n, m}\left(\alpha_{l, n}\right)\right)\right] \bar{B}_{l, n, m} \\
& \frac{\partial U}{\partial r_{z}}=\frac{\mu}{R_{b}} \sum_{l=0}^{l_{\max }} \sum_{n=0}^{n_{\max }} \sum_{m=0}^{n} R e\left[\frac{\partial}{\partial z}\left(\bar{\beta}_{n, m}\left(\alpha_{l, n}\right)\right)\right] \bar{A}_{l, n, m}+\operatorname{Im}\left[\frac{\partial}{\partial z}\left(\bar{\beta}_{n, m}\left(\alpha_{l, n}\right)\right)\right] \bar{B}_{l, n, m}
\end{aligned}
$$

- Basis Functions: $\bar{\beta}_{n, m}\left(\alpha_{l, n}\right)=j_{n}\left[\frac{\alpha_{l, n} r}{R_{b}}\right] \bar{H}_{n, m}$

$$
\bar{H}_{n, m}= \begin{cases}N_{n, m} P_{n, m}[\sin (\phi)] e^{i m \lambda} & n \geq m \geq 0 \\ 0 & \text { otherwise }\end{cases}
$$

- Partials of the Basis Functions:

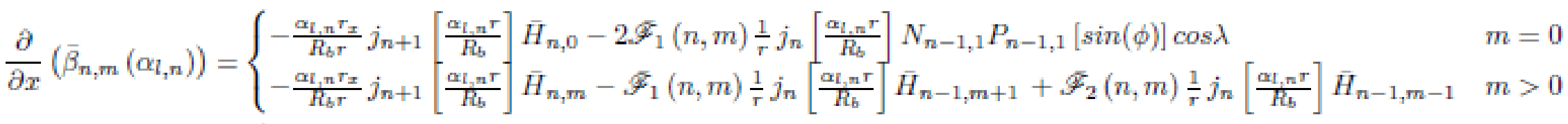

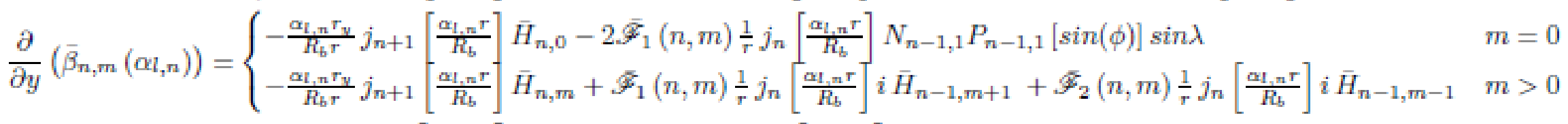

$$
\begin{aligned}
& \frac{\partial}{\partial z}\left(\bar{\beta}_{n, m}\left(\alpha_{l, n}\right)\right)=-\frac{\alpha_{l, n} r_{z}}{R_{b} r} j_{n+1}\left[\frac{\alpha_{l, n} r}{R_{b}}\right] \bar{H}_{n, m}+\bar{F}_{3}(n, m) \frac{1}{r} j_{n}\left[\frac{\alpha_{l, n} r}{R_{b}}\right] \bar{H}_{n-1, m}
\end{aligned}
$$




\section{Thrust Profile}

- Three classes of thrust profiles

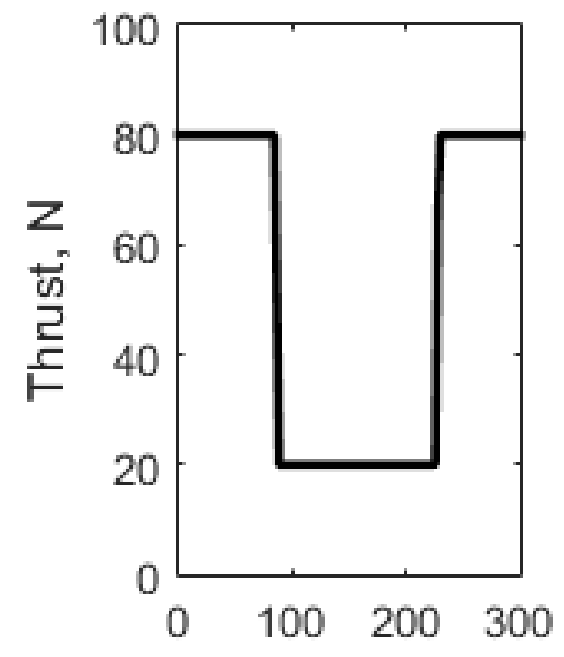

a) Time, $s$

a) $300 \mathrm{~s}$,

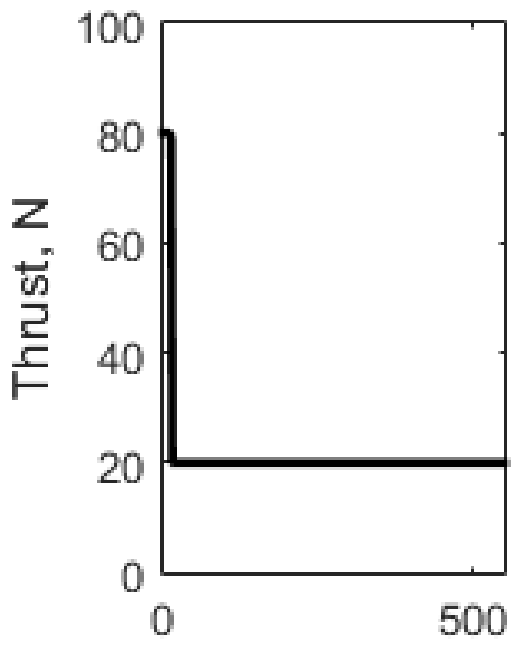

b) Time, $s$

b) $550 \mathrm{~s}$,

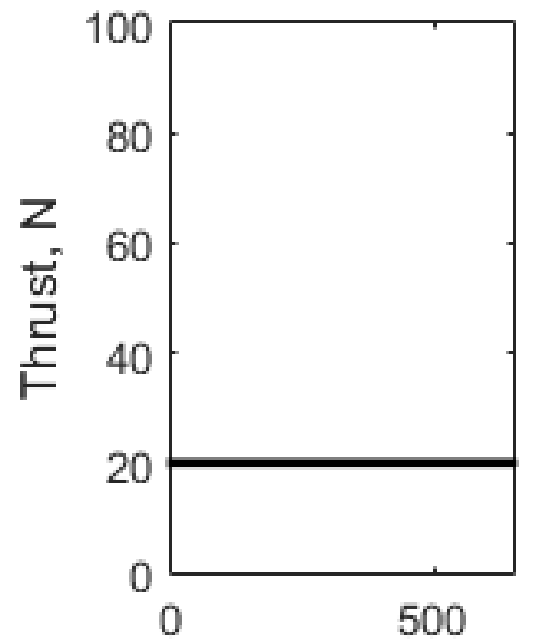

c) Time, $s$

c) $650 \mathrm{~s}$ 